\title{
SYSTÈMES DOUBLEMENT ORTHOGONAUX DE FONCTIONS HOLOMORPHES ET APPLICATIONS
}

\author{
THANH VAN NGUYEN et AHMED ZERIAHI \\ Laboratoire d'Analyse Complexe et Fonctionnelle, Université Paul Sabatier \\ 118, Route de Narbonne, F-31062 Toulouse Cedex, France
}

0. Introduction. Nous donnons ici une étude systématique des systèmes doublement orthogonaux "de Bergman" et leurs applications à certains aspects de l'analyse pluricomplexe : espaces de fonctions holomorphes, fonctions séparément analytiques.

C'est en quelque sorte un article de synthèse. On y trouve cependant des démonstrations détaillées qui n'ont paru nulle part ailleurs.

1. Fonctions plurisousharmoniques extrémales et mesures d'équilibre. Soit $D$ un ouvert de $\mathbb{C}^{n}$ et $E \subset \mathbb{C}^{n}$. On définit la fonction plurisousharmonique extrémale associée au couple $(E, D)([\mathrm{SC}, 2])$ par

$$
\omega(z, E, D)=\limsup _{z^{\prime} \rightarrow z}\left[\sup \left\{u\left(z^{\prime}\right): u \in P_{01}(E, D)\right\}\right], \quad z \in D,
$$

où $P_{0,1}(E, D)$ est la classe des fonctions $u$ plurisousharmoniques sur $D$ telles que $u \leq 1$ et $u / E \cap D \leq 0$. Elle vérifie l'équation de Monge-Ampère complexe ([B-T], $[\mathrm{K}])$ :

$$
\left(d d^{c} \omega(\cdot, E, D)\right)^{n}=0 \quad \text { sur } D \backslash \bar{E} .
$$

Pour $\alpha \in[0,1[$ on pose

$$
D_{\alpha}=D(E, \alpha):=\{z \in D: \omega(Z, E, D)<\alpha\}
$$

Si $D$ est borné, alors

$$
\omega\left(z, E, D_{\alpha}\right)=\frac{1}{\alpha} \omega(z, E, D), \quad \forall z \in D_{\alpha}
$$

1991 Mathematics Subject Classification: 32A05, 32A37, 32D10.

Key words and phrases: extremal functions, doubly orthogonal systems, Schauder bases, separately analytic functions.

The paper is in final form and no version of it will be published elsewhere. 
(1.4bis) $E$ est "localement" non pluripolaire dans $D_{\alpha}$, c'est-à-dire $E$ rencontre chaque composante connexe de $D_{\alpha}$ en un ensemble non pluripolaire (voir p. ex. [N-Z 2, Lemme 3], voir également la proposition 1.2 cidessous).

On appelle condensateur dans $\mathbb{C}^{n}$ tout couple $(K, D)$ formé d'un ouvert hyperconvexe $D$ de $\mathbb{C}^{n}$ et d'un compact $K \subset D$ "localement" non pluripolaire dans $D$. On rappelle qu'on ouvert $D$ de $\mathbb{C}^{n}$ est dit hyperconvexe lorsqu'il existe une fonction plurisousharmonique $\varrho$ sur $D, \varrho<0$ et telle que $\{z \in D: \varrho(z)<c\} \Subset D$ pour tout $c<0$. Une telle $\varrho$ est appellée une fonction plurisousharmonique d'exhaustion bornée de $D$.

Pour tout condensateur $(K, D)$, le courant positif $\left(d d^{c} \omega(\cdot, K, D)\right)^{n}$ s'identifie à une mesure positive portée par $K$. Nous la noterons $\mu_{0}(K, D)$ ou $\mu_{0}$ tout simplement et nous l'appellerons mesure d'équilibre du condensateur $(K, D)$.

Un condensateur $(K, D)$ est dit régulier, ou P-régulier, lorsque $\omega(\cdot, K, D)$ $=0$ sur $K$. On sait qu'alors $\omega(\cdot, K, D)-1$ est une fonction plurisousharmonique d'exhaustion bornée continue de $D$ et (1.4) est vérifiée [Za,1].

Proposition 1.1. Soit $(K, D)$ un condensateur dans $\mathbb{C}^{n}, \mu_{0}$ sa mesure d'équilibre. Alors si $E \subset K$ est $\mu_{0}$-mesurable et $\mu_{0}(E)=\mu_{0}(K)$, on $a \omega(\cdot, E, D)=$ $\omega(\cdot, K, D)$.

Ce résultat est prouvé dans $[\mathrm{N}-\mathrm{Z}, 1]$ dans le cas où $D$ est borné. Pour le cas général, voir [Ze,2].

Proposition 1.2. Soit $(K, D)$ un condensateur, $\mu_{0}$ sa mesure d'équilibre.

1) Si $u$ est plurisousharmonique sur $D$ et $u=-\infty \mu_{0}$-presque partout sur $K$, alors $u \equiv-\infty$.

2) Si $(K, D)$ est régulier, alors 1$)$ est vraie avec $D_{\alpha}$ à la place de $\left.D, \forall \alpha \in\right] 0,1[$.

Démonstration. On se contente de prouver 2), l'autre preuve étant semblable. Soit $\beta \in] 0, \alpha\left[\right.$; alors $D_{\beta} \Subset D_{\alpha}$ et $u$ est donc majorée sur $\bar{D}_{\beta}$ par un certain $M \in \mathbb{R}$. Pour tout $A>0$ on a $u+A \leq M+A$ sur $D_{\beta}$ et $u+A \leq 0 \mu_{0}$-presque partout sur $K$. Il existe donc $E \subset K, \mu_{0}$-mesurable tel que $\mu_{0}(E)=\mu_{0}(K)$ et $u+A \leq 0$ sur $E$. Par suite,

$$
u \leq M \omega\left(\cdot, E, D_{\beta}\right)+A\left(\omega\left(\cdot, E, D_{\beta}\right)-1\right) .
$$

D'après la proposition 1.1 et (1.4),

$$
\omega\left(\cdot, E, D_{\beta}\right)=\frac{1}{\beta} \omega(\cdot, E, D)=\frac{1}{\beta} \omega(\cdot, E, D)=\frac{1}{\beta} \omega(\cdot, E, D)<1 .
$$

En faisant tendre $A$ vers $\infty$, on obtient $u \equiv-\infty$ sur $D_{\beta}$.

2. Systèmes doublement orthogonaux de Bergman. Nous aurons besoin d'un théorème d'approximation dont la démonstration utilise les estimations $L^{2}$ do Hörmander [Hö]. 
Lorsque $D$ est un ouvert de $\mathbb{C}^{n}$, on désigne par $\mathcal{O}(D)$ l'espace des fonctions holomorphes sur $D$ muni de la topologie de la convergence compacte sur $D$. Lorsque $K$ est un compact de $\mathbb{C}^{n}$, on désigne par $\mathcal{O}(K)$ l'espace des germes de fonctions holomorphes au voisinage de $K$ muni de sa topologie $\mathcal{L} \mathcal{F}$.

Soit $\lambda$ la mesure de Lebesgue dans $\mathbb{C}^{n}$, et $\mu_{1}$ la mesure définie par $d \mu_{1}=$ $\left(1+|z|^{2}\right)^{-2} d \lambda$. Désignons par $\mathcal{O}^{2}\left(D, d \mu_{1}\right)$ l'espace de Hilbert des fonctions holomorphes sur $D$ de carré $\mu_{1}$-intégrable sur $D$.

ThÉORÈme 2.1. Soit $D$ un ouvert hyperconvexe de $\mathbb{C}^{n}$. Alors l'espace $\mathcal{O}^{2}\left(D, d \mu_{1}\right)$ est dense dans l'espace $\mathcal{O}(D)$.

Démonstration. Soit $E$ un compact de $D$ et $\varrho: D \rightarrow] 0,1[$ une fonction plurisousharmonique exhaustive sur $D$. Soit $a, b \in] 0,1\left[\right.$ tels que $\sup _{E} \varrho<a<$ $b<1$, de sorte que $\varphi:=\varrho-a$ est plurisousharmonique sur $D, \varphi<0$ sur $D_{a}:=\{z \in D: \varrho(z)<a\}$ et $\varphi \geq b-a>0$ sur $D \backslash D_{b}$.

Soit $\chi$ une fonction $C^{\infty}$ à support compact dans $D$ telle que $\chi \equiv 1$ sur $D_{b}$. Soit $f \in \mathcal{O}(D)$; alors $\chi f \in C^{\infty}(D) \cap L^{2}\left(D, d \mu_{1}\right)$ et $\chi f=f$ sur $D_{b}$. Nous allons corriger cette fonction pour la rendre holomorphe. On cherche une fonction $u$ de la même classe que $\chi f$ telle que la fonction

$$
g=\chi f-u
$$

soit holomorphe sur $D$ et $u$ soit "assez petite" sur $E$. Cela se fait classiquement par le théorème d'existence de Hörmander pour un choix convenable des fonctions poids. Pour chaque entier $k \geq 1$, soit $\varphi_{k}=k \varphi$. Il est clair que $|f \bar{\partial} \chi|^{2} e^{-k \varphi}$ est $\lambda$-intégrable sur $D$. D'après le théorème de Hörmander il existe $u_{k}$ localement intégrable sur $D$ telle que

$$
\begin{gathered}
\bar{\partial} u_{k}=f \bar{\partial}_{\chi} \quad \text { sur } D, \\
\int_{D}\left|u_{k}\right|^{2} e^{-\tilde{\varphi}_{k}} d \lambda \leq \int_{D}\left|f \bar{\partial}_{\chi}\right|^{2} e^{-\varphi_{k}} d \lambda
\end{gathered}
$$

où $\widetilde{\varphi}_{k}=\varphi_{k}+2 \log \left(1+|z|^{2}\right)$.

Comme $\bar{\partial}_{\chi}=0$ sur $D_{b}$ et $\varrho \geq b-a=: \varepsilon>0$ sur $D \backslash D_{b}$, le second membre de (2.3) est $O\left(e^{-k \varepsilon}\right)$ lorsque $k \rightarrow \infty$. Puisque $\varphi<a$ sur $D a \Subset D$, il résulte de $(2.3)$ qu'il existe une constante $C>0$ telle que

$$
\int_{D}\left|u_{k}\right|^{2} d \lambda \leq C e, \quad \forall k \geq 1
$$

D'après (1.2) et le fait que $\chi \equiv 1$ sur $D_{b}$, on en déduit que $u_{k}$ est holomorphe sur $D_{b} \supset D_{a}$. Il résulte alors de l'inégalité de la moyenne et de (2.4) que la suite $\left(u_{k}\right)$ tend vers 0 uniformément sur tout compact de $D_{a}$. Par conséquent, la suite $g_{k}:=\chi f-u_{k}$ est une suite de fonctions holomorphes sur $D$ qui converge uniformément sur tout compact de $D_{a}$, donc sur $E$, vers $f$. Comme $\varphi<1-a$ sur $D$, l'inégalité $(2.3)$ montre que $u_{k} \in L^{2}\left(D, d \mu_{1}\right)$. Par conséquent, $g_{k} \in \mathcal{O}^{2}\left(D, d \mu_{1}\right)$ 
et $g_{k} \rightarrow f$ uniformément sur $E$. Comme $E$ est un compact quelconque de $D$, le théorème en résulte.

Remarque. Ce théorème nous sera utile au paragraphe 4, on en déduit que $\mathcal{O}^{2}\left(D, d \mu_{1}\right)$ est de dimension infinie. C'est ce fait qui sera utilisé dans la construction suivante. Rappelons que $\mu_{1}$ est la mesure borélienne sur $D$ définie par $d \mu_{1}=\left(1+|z|^{2}\right)^{-2} d \lambda$.

Soit $(K, D)$ un condensateur de $\mathbb{C}^{n}$. Nous allons construire un système doublement orthogonal par la méthode classique de Bergman qui est un cas particulier de la construction des fonctions propres d'un opérateur compact autoadjoint et positif d'un espace de Hilbert.

Soit $H_{1}=\mathcal{O}^{2}\left(D, d \mu_{1}\right)$ et $H_{0}$ le sous-espace vectoriel fermé de $L^{2}\left(K, d \mu_{0}\right)$ engendré par la restriction à $K$ des fonctions de $H_{1}$. On a alors une injection continue à image dense

$$
i: H_{1} \rightarrow H_{0} \text {. }
$$

Posons $A=i^{*} \circ i$. Alors $A$ est un opérateur linéaire compact autoadjoint et positif vérifiant

$$
(A f \mid g)_{1}=(f \mid g)_{0} \quad \forall f, g \in H_{1} .
$$

On a identifié $f$ à $i(f)$ qui est la restriction de $f$ à $K,(\cdot \mid \cdot)_{k}$ désignant le produit scalaire sur $H_{k}, k=0,1 \ldots$

D'après (2.6), $A$ est injectif. On sait qu'alors $A$ possède une suite $\left(\lambda_{j}\right)_{j \geq 1}$ de valeurs propres $>0$ et $H_{1}$ possède une base orthonormée $\left(\varphi_{j}\right)_{j \geq 1}$ de fonctions propres $([\mathrm{R}-\mathrm{N}])$. Rappelons la construction classique de cette base. Par un argument de compacité on montre que

$$
\lambda_{1}:=\sup _{\|f\|_{0}=1}\left|(A f \mid f)_{1}\right|=\sup _{\|f\|_{0}=1}\|f\|_{0}^{2}
$$

est atteint en un point $\psi_{1} \in H_{1}$ tel que $A\left(\psi_{1}\right)=\lambda_{1} \psi_{1}$. En raisonnant par récurrence sur $p \geq 1$, supposons $\psi_{1}, \ldots, \psi_{p}$ construits et soit $H_{p+1}$ le sous-espace fermé de $H_{1}$, orthogonal au système $\left\{\psi_{1}, \ldots, \psi_{p}\right\}$. Alors on a $A\left(H_{p+1}\right) \subset H_{p+1}$ et on peut donc itérer la construction précédente pour obtenir un élément $\psi_{p+1}$ tel que

$$
\begin{gathered}
\lambda_{p+1}:=\sup \left\{(A f \mid f)_{1}: f \in H_{p+1},|f|_{1}=1\right\}=\left\|\psi_{p+1}\right\|_{0}, \\
A\left(\psi_{p+1}\right)=\lambda_{p+1} \psi_{p+1}, \quad \psi_{p+1} \in H_{p+1},\left\|\psi_{p+1}\right\|_{1}=1 .
\end{gathered}
$$

Il est alors clair que $\left(\psi_{p}\right)$ est un système orthonormé de $H_{1}$ orthogonal dans $H_{0}$.

Puisque $A$ est injectif, 0 n'est pas valeur propre de $A$ et la décomposition spectrale de $A$ montre que $\left\{\psi_{p}\right\}$ est une base orthonormée de $H_{1}$. Posons

$$
\begin{aligned}
\varphi_{p} & =\lambda_{p}^{-1} \psi_{p}, \\
\gamma_{p} & =\lambda_{p}^{-1} .
\end{aligned}
$$

Alors $\left(\varphi_{p}\right)$ est une base orthogonale de $H_{1}$, orthonormée dans $H_{0}$. 
Cette construction a été faite par Bergman dans le cas d'une variable complexe ([Be]). Suivant sa terminologie, nous appellerons $\left(\varphi_{p}\right)$ le système doublement orthogonal de Bergman associé au condensateur $(K, D)$ (en abrégé : S.D.O.B.).

Il résulte de la construction précédente que $\left(\gamma_{p}\right)$ est une suite croissante tendant vers $\infty$, qui ne dépend que du condensateur $(K, D)$. Il est possible de l'interpréter en terme de $n$-diamètre de Kolmogorov et d'après Mityagin [Mi], la nucléarité de l'espace $O(D)$ implique la propriété suivante :

$$
\sum_{j=1}^{\infty} \gamma_{j}^{-\varepsilon}<\infty, \quad \forall \varepsilon>0
$$

Grâce à cette propriété, on démontre le résultat suivant (voir [N-Z,2], [N-S]) :

Proposition 2.2. Le S.D.O.B. associé au condensateur $(K, D)$ vérifie l'estimation suivante:

$$
\limsup _{j \rightarrow \infty} \frac{\log \left|\varphi_{j}(z)\right|}{\log \gamma_{j}} \leq \omega(z ; K, D), \quad \forall z \in D .
$$

Grâce au lemme de Hartogs classique, il en résulte facilement :

Corollaire 2.3. Pour tout $\alpha \in] 0,1\left[\right.$, et tout compact $E \subset D_{\alpha}$, il existe une constante $C>0$ telle que

$$
\left|\varphi_{j}\right|_{E}:=\sup _{z \in E}\left|\varphi_{j}(z)\right| \leq C \gamma_{j}^{\alpha}, \quad \forall j \in N^{*}
$$

3. Convexité logarithmique des normes duales sur l'espace $\mathcal{O}^{\prime}(D)$. Au paragraphe 2 , nous avons obtenu une estimation du système $\left(\varphi_{j}\right)$. Ici nous voulons donner une estimation du système biorthogonal $\left(\varphi_{j}^{\prime}\right)$ associé à $\left(\varphi_{j}\right)$. Posons

$$
\|f\|_{\alpha}:=\left(\int_{D_{\alpha}}|f|^{2} d \mu_{1}\right)^{1 / 2}, \quad 0<\alpha \leq 1, f \in \mathcal{O}(D) .
$$

D'après l'inégalité de la moyenne, les normes (3.1) engendrent la topologie de $\mathcal{O}(D)$. Pour $\alpha=0$ rappelons que $\|f\|_{0}^{2}=\int_{K}|f|^{2} d \mu_{0}$.

On définit les normes duales de $(3.1)$ sur $\mathcal{O}^{\prime}(D)$ :

$$
\|T\|_{\alpha}^{*}:=\sup \left\{\mid\langle T, f\rangle: f \in \mathcal{O}(D),\|f\|_{\alpha} \leq 1\right\}, \quad T \in \mathcal{O}^{\prime}(D) .
$$

On suppose dans la suite que $(K, D)$ est régulier.

ThÉorème 3.1. Soient $\left.0 \leq \alpha_{0} \leq \alpha_{1} \leq 1, \theta \in\right] 0,1[, \varepsilon \in] 0, \theta[$. Alors il existe une constante $C_{1}>0$ (dépendant de $\alpha_{0}, \alpha_{1}, \theta, \varepsilon$ ) telle que

$$
\|T\|_{(1-\theta) \alpha_{0}+\theta \alpha_{1}}^{*} \leq C_{1}\|T\|_{\alpha_{0}}^{* 1-\theta+\varepsilon}\|T\|_{\alpha_{1}}^{* \theta-\varepsilon}, \quad \forall T \in \mathcal{O}^{\prime}(D) .
$$


La démonstration de ce théorème nécessite quelques préliminaires. On considère dans $\mathcal{O}(D)$ les boules associées aux normes (3.1) :

$$
\begin{aligned}
& \mathcal{U}_{\alpha}:=\left\{f \in \mathcal{O}(D): \int_{D_{\alpha}}|f|^{2} d \mu \leq 1\right\}, \quad 0<\alpha \leq 1, \\
& \mathcal{U}_{0}:=\left\{f \in \mathcal{O}(D): \int_{D_{k}}|f|^{2} d \mu_{0} \leq 1\right\} .
\end{aligned}
$$

Le théorème 3.1 sera une conséquence du résultat suivant :

Proposition 3.2. Soient $0 \leq \alpha_{0}<\alpha_{1} \leq 1,0<\theta<1$. Alors pour tout $\beta \in] 0,1\left[\right.$ tel que $\beta>(1-\theta) \alpha_{0}+\theta \alpha_{1}$, il existe une constante $C_{2}>0$ telle que

$$
\mathcal{U}_{\alpha} \subset C_{2} r^{-\theta} \mathcal{U}_{\alpha_{0}}+C_{2} r^{1-\theta} U_{\alpha_{1}}, \quad \forall r>0 .
$$

Montrons tout d'abord que la proposition 3.2 implique le théorème 3.1.

Démonstration du théorème 3.1. Soit $T \in \mathcal{O}^{\prime}(D), \alpha_{0}, \alpha_{1} \in[0,1]$ tels que $\left.0 \leq \alpha_{0}<\alpha_{1} \leq 1, \theta \in\right] 0,1\left[, \beta \in[0,1]\right.$ tel que $\beta>(1-\theta) \alpha_{0}+\theta \alpha_{1}$. Soit $f \in \mathcal{U}_{\beta}$ et $r>0$. D'après la proposition 3.2, il existe $g \in C_{2} r^{-\theta} \mathcal{U}_{\alpha_{0}}$ et $h \in C_{2} r^{1-\theta} \mathcal{U}_{\alpha_{1}}$ tels que $f=g+h$ sur $D$. Il en résulte alors que

$$
|T(f)| \leq|T(g)|+|T(h)| \leq C_{2}\left(r^{-\theta}\|T\|_{\alpha_{0}}^{*}+r^{1-\theta}\|T\|_{\alpha_{1}}^{*}\right),
$$

ce qui implique l'inégalité suivante :

$$
\|T\|_{\beta}^{*} \leq C_{2}\left(r^{-\theta}\|T\|_{\alpha_{0}}^{*}+r^{1-\theta}\|T\|_{\alpha_{1}}^{*}\right), \quad \forall r>0 .
$$

La borne inférieure du second membre lorsque $r>0$ varie est égale à

$$
C_{2}(1-\theta)^{\theta-1} \theta^{-\theta}|T|_{\alpha_{0}}^{* 1-\theta}|T|_{\alpha_{0}}^{* \theta}
$$

On en déduit immédiatement les estimations (3.3) en remplaçant $\theta$ par $\theta-\varepsilon$ et $\beta=(1-\theta) \alpha_{0}+\theta \alpha_{1}>(1-\theta+\varepsilon) \alpha_{0}+\theta \alpha_{1}>(1-\theta+\varepsilon) \alpha_{0}+(\theta-\varepsilon) \alpha_{1}$

Démonstration de la proposition 3.2. Nous aurons besoin du lemme suivant tout à fait classique qui se démontre modulo une partition de l'unité et estimations $L^{2}$ de Hörmander (voir $[\mathrm{A}]$ ).

LEMme 3.3. Soit $\Omega$ un ouvert pseudoconvexe de $\mathbb{C}^{n},\left\{\Omega^{+}, \Omega^{-}\right\}$un recouvrement de $\Omega$ tel que $\Omega^{+} \cap \Omega^{-} \Subset \Omega$. Alors il existe une constante $M>0$ telle que pour toute fonction $v$ p.s.h. sur $\Omega$ et pour tout $f \in \mathcal{O}(\Omega)$ il existe $f^{+} \in \mathcal{O}\left(\Omega^{+}\right)$, $f^{-} \in \mathcal{O}\left(\Omega^{-}\right)$vérifiant :

(i) $f=f^{+}-f^{-} \operatorname{sur} \Omega^{+} \cap \Omega^{-}$,

(ii) $\int_{\Omega^{ \pm}}\left|f^{ \pm}\right|^{2} e^{-\tilde{v}} d \lambda \leq M \int_{\Omega^{+} \cap \Omega^{-}}|f|^{2} e^{-v} d \lambda$

ò̀ $\widetilde{v}(z)=v(z)+2 \log \left(1+|z|^{2}\right)$.

Soient $\alpha_{0}, \alpha_{1}, \alpha, \beta \in[0,1]$ tels que $0<\alpha_{0}<\alpha \leq \beta<\alpha_{1} \leq 1$. On notera pour simplifier $\omega=\omega(0, K, D)$. 
Posons $\Omega=D, \Omega^{+}=D_{\beta}, \Omega^{-}=D \backslash \bar{D}_{\alpha}$. Soit $\varrho \geq 0$. Posons

$$
v=v_{p}:=\varrho \frac{\left(\omega-\alpha_{0}\right)^{+}}{\alpha_{1}-\alpha_{0}}
$$

où $\left(\omega-\alpha_{0}\right)^{+}=\sup \left\{\omega-\alpha_{0}, 0\right\}$. Appliquons alors le lemme 3.3.

Soit $f \in U_{\beta}$. On a, en posant $B_{1}=\sup _{z \in D_{\beta}}\left(1+|z|^{2}\right)$,

$$
\int_{\Omega^{+} \cap \Omega^{-}}|f|^{2} e^{-v} d \lambda \leq B_{1} \sup _{\Omega^{+} \cap \Omega^{-}} e^{-v} \leq B_{1} \exp \left(-\frac{\alpha-\alpha_{0}}{\alpha_{1}-\alpha_{0}} \varrho\right) .
$$

D'après le lemme 3.3 , il existe $f^{ \pm} \in \mathcal{O}\left(\Omega^{ \pm}\right)$telle que

$$
f=f^{+}-f^{-} \quad \text { sur } \Omega^{+} \cap \Omega^{-}=D_{\beta} \backslash \bar{D}_{\alpha} .
$$

Compte tenu de l'estimation (3.5), les inégalités (ii) du lemme s'écrivent, en posant $B_{2}=B_{1} M$

$$
\begin{gathered}
\int_{D_{\beta}}\left|f^{+}\right|^{2} e^{-\tilde{v}} d \lambda \leq B_{2} \exp \left(-\frac{\alpha-\alpha_{0}}{\alpha_{1}-\alpha_{0}} \varrho\right), \\
\int_{D \backslash \bar{D}_{\alpha}}\left|f^{-}\right|^{2} e^{-\tilde{v}} d \lambda \leq B_{2} \exp \left(-\frac{\alpha-\alpha_{0}}{\alpha_{1}-\alpha_{0}} \varrho\right)
\end{gathered}
$$

Posons maintenant

$$
g:= \begin{cases}f^{+} & \text {sur } D^{+}=D_{\beta}, \\ f+f^{-} & \text {sur } D^{-}=D \backslash \bar{D}_{\alpha} .\end{cases}
$$

D'après la relation (3.6), $g$ est bien défini et donc $g \in \mathcal{O}(D), h:=f-g \in \mathcal{O}(D)$ et l'on a

$$
f=g+h \quad \text { sur } D .
$$

Nous allons prouver les estimations suivantes :

$$
\begin{gathered}
\int_{D_{\alpha_{0}}}|g|^{2} d \mu_{1} \leq C_{2} \exp \left(-\frac{\alpha-\alpha_{0}}{\alpha_{1}-\alpha_{0}} \varrho\right), \\
\int_{D_{\alpha_{1}}}|h|^{2} d \mu_{1} \leq C_{2} \exp \left(\frac{\alpha_{1}-\alpha}{\alpha_{1}-\alpha_{0}} \varrho\right),
\end{gathered}
$$

où $C_{2}$ est une constante qui ne dépend ni de $f$, ni de $\varrho$.

Commençons par prouver (3.12). Par définition $h=f-g$. Compte tenu de (3.9), on a alors

$$
\int_{D_{\alpha_{1}}}|h|^{2} d \mu_{1}=\int_{D_{\alpha_{1}} \backslash \bar{D}_{\alpha}}\left|f^{-}\right|^{2} d \mu_{1}+\int_{\bar{D}_{\alpha}}\left|f-f^{+}\right|^{2} d \mu_{1}
$$


D'après (3.8) et le fait que $v \leq \varrho$ sur $D_{\alpha_{1}}$, on obtient

$$
\int_{D_{\alpha_{1}} \backslash \bar{D}_{\alpha}}\left|f^{-}\right|^{2} d \mu_{1} \leq \sup _{D_{\alpha_{1}}} e^{v} \int_{D_{\alpha_{1}} \backslash \bar{D}_{\alpha}}\left|f^{-}\right|^{2} e^{-\tilde{v}} d \lambda \leq B_{2} \exp \left(\frac{\alpha_{1}-\alpha}{\alpha_{1}-\alpha_{0}} \varrho\right) .
$$

D'autre part, on a

$$
\left.\left(\int_{\bar{D}_{\alpha_{1}}}\left|f-f^{+}\right|^{2} d \mu_{1}\right)^{1 / 2} \leq\left(\int_{\bar{D}_{\alpha_{1}}}|f|^{2} d \mu_{1}\right)^{1 / 2}+\underset{\bar{D}_{\alpha_{1}}}{\int}\left|f^{+}\right|^{2} d \mu_{1}\right)^{1 / 2} .
$$

D'après (3.7) et le fait que $v \leq \varrho$ sur $\bar{D}_{\alpha} \subset D_{\alpha_{1}}$, on a

$$
\int_{\bar{D}_{\alpha_{1}}}|f|^{2} d \mu_{1} \leq \sup _{\bar{D}_{\alpha}} e^{v} \int_{\bar{D}_{\alpha_{1}}}\left|f^{+}\right|^{2} e^{-\tilde{v}} d \lambda \leq B_{2} \exp \left(\frac{\alpha_{1}-\alpha}{\alpha_{1}-\alpha_{0}} \varrho\right) .
$$

Comme $f \in \mathcal{U}_{\beta}$ et que $D_{\alpha} \subset D_{\beta}$, il résulte des estimations (3.15) et (3.16) que l'on a

$$
\left(\int_{\bar{D}_{\alpha_{1}}}\left|f-f^{+}\right|^{2} d \mu_{1}\right)^{1 / 2} \leq\left(1+B_{2}\right) \exp \left(\frac{\alpha_{1}-\alpha}{\alpha_{1}-\alpha_{0}} \varrho\right) .
$$

L'estimation (3.12) résulte clairement des estimations (3.13), (3.14) et (3.17) en posant $C_{2}=1+2 B_{2}$.

Il reste à prouver (3.11). En effet, d'après (3.9), on a

$$
\int_{D_{\alpha_{1}}}|g|^{2} d \mu_{1} \leq \int_{D_{\alpha_{0}}}\left|f^{+}\right|^{2} d \mu_{1} \leq \sup _{D_{\alpha_{0}}} e^{v} \int_{D_{\alpha_{0}}}\left|f^{+}\right|^{2} e^{-v} d \mu_{1}
$$

Comme $v=0$ sur $D_{\alpha_{0}}$, on en déduit d'après (3.7),

$$
\int_{D_{\alpha_{0}}}|g|^{2} d \mu_{1} \leq B_{2} \exp \left(-\frac{\alpha-\alpha_{0}}{\alpha_{1}-\alpha_{0}}\right) \text {. }
$$

On en déduit (3.11) avec $C_{2}=1+2 B_{2}$ puisque $B_{2} \leq C_{2}$.

Soit maintenant $\theta \in] 0,1\left[\right.$. Choisissons $\alpha:=(1-\theta) \alpha_{0}-\theta \alpha_{1}$, de sorte que

$$
\frac{\alpha-\alpha_{0}}{\alpha_{1}-\alpha_{0}}=\theta \quad \text { et } \quad \frac{\alpha_{1}-\alpha}{\alpha_{1}-\alpha_{0}}=1-\theta
$$

Alors pour tout $\beta$ tel que $(1-\theta) \alpha_{0}+\theta \alpha_{1}<\beta<\alpha_{1}$ et $f \in \mathcal{U}_{\beta}$, on a la décomposition (3.10) avec les estimations (3.11) et (3.12) qui signifient que $g \in C_{1} r^{-\theta} \mathcal{U}_{\alpha_{0}}$ et $h \in C_{1} r^{1-\theta} \mathcal{U}_{\alpha_{1}}$, avec $r=e^{\varrho}$. Cela prouve donc les inclusions (3.4) pour $r>1$ et $\alpha_{0}>0$.

Si $r \in] 0,1]$ et $\alpha_{0}>0$, on a $r^{-\theta} \geq 1$ et donc $\mathcal{U}_{\beta} \subset \mathcal{U}_{\alpha_{0}} \subset r^{-\theta} \mathcal{U}_{\alpha_{0}} \subset C_{2} r^{-\theta} \mathcal{U}_{\alpha_{0}}$, ce qui prouve (3.4) dans ce cas.

Il reste à montrer que (3.4) reste valable si $\alpha_{0}=0$. Pour cela il suffit d'observer que si $\beta>\theta \alpha_{1}$, il existe $\varepsilon>0$ tel que $\beta>(1-\theta) \varepsilon+\theta \alpha_{1}$ et donc d'après ce qui précède on a $\mathcal{U}_{\beta} \subset C_{2} r^{-\theta} \mathcal{U}_{\varepsilon}+C_{2} r^{1-\theta} \mathcal{U}_{\alpha_{1}}, \forall r>0$. 
D'après l'inégalité de la moyenne, il existe une constante $C(\varepsilon)>0$ telle que

$$
\sup _{K}|g|^{2} \leq C(\varepsilon) \int_{D_{\varepsilon}}|g|^{2} d \mu_{1}, \quad \forall g \in \mathcal{O}(D) .
$$

Par conséquent, on a

$$
\int_{K}|d g|^{2} d \mu_{0} \leq \mu_{0}(K) C(\varepsilon) \int_{D_{\varepsilon}}|g|^{2} d \mu_{1},
$$

ce qui prouve que $\mathcal{U}_{\varepsilon} \subset C^{\prime}(\varepsilon) \mathcal{U}_{0}$ où $C^{\prime}(\varepsilon)=\mu_{0}(K) C(\varepsilon)$.

On en déduit que $\mathcal{U}_{\beta} \subset C_{2}^{\prime} r^{-\theta} \mathcal{U}_{0}+C_{2} r^{1-\theta} \mathcal{U}_{\alpha_{1}}, \forall r>0$, ce qui prouve encore (3.4) pour la constante $\widetilde{C}_{2}=\max \left\{C_{2}^{\prime}, C_{2}\right\}$.

Les inégalités de convexité du théorème 4.1 constituent une version très précise de la propriété $(\bar{\Omega})$ de Vogt $[\mathrm{V}]$ pour l'espace de Fréchet $\mathcal{O}(D)$. Cette propriété $(\bar{\Omega})$ a été établie par Aytuna $([\mathrm{A}])$ pour l'espace $\mathcal{O}(D)$ en démontrant une version plus faible de la proposition 4.2. Sa méthode est basée sur les estimations $L^{2}$ de Hörmander $[\mathrm{H}]$; c'est cette méthode que nous avons reprise ici $([\mathrm{Ze}, 1])$.

\section{S.D.O.B. et bases de Schauder communes des espaces $\mathcal{O}(D)$ et $\mathcal{O}\left(\widehat{K}_{D}\right)$}

ThÉorème 4.1. Soit $(K, D)$ un condensateur régulier dans $\mathbb{C}^{n}$. Soit $\left(\varphi_{j}\right)$ le S.D.O.B. associé à ce condensateur. Alors :

1) $\left(\varphi_{j}\right)$ est une base de Schauder des espaces $\mathcal{O}\left(D_{\alpha}\right), \mathcal{O}(D)$ et $\mathcal{O}\left(\widehat{K}_{D}\right), \widehat{K}_{D}$ désignant l'enveloppe holomorphe-convexe de $K$ dans $D$ et $D_{\alpha}=D(K, \alpha)$, $0<\alpha<1$.

2) Pour tout $\alpha \in] 0,1\left[\right.$ et toute suite complexe $\left(c_{j}\right)$, les propriétés suivantes sont équivalentes :

(i) $\sum c_{j} \varphi_{j}$ converge dans $\mathcal{O}\left(D_{\alpha}\right)$,

(ii) $\limsup _{j \rightarrow \infty} \frac{\log \left|c_{j}\right|}{\log \gamma_{j}} \leq-\alpha$.

3) $\left.\lim _{j \rightarrow \infty} \frac{\log \left|\varphi_{j}\right|_{D_{\alpha}}}{\log \gamma_{j}}=\alpha, \quad \forall \alpha \in\right] 0,1[$.

4) Reg sup $\left(\limsup _{j \rightarrow \infty} \frac{\log \left|\varphi_{j}\right|}{\log \gamma_{j}}\right)=\omega(\cdot, K, D)$ sur $D \backslash \widehat{K}_{D}$.

Démonstration. 1) Il suffit de prouver que $\left(\varphi_{j}\right)$ est une base de $\mathcal{O}\left(D_{\alpha}\right)$, car

$$
\mathcal{O}(D)==\lim _{\alpha \uparrow 1} \operatorname{proj} \mathcal{O}\left(D_{\alpha}\right), \quad \mathcal{O}\left(\widehat{K}_{D}\right)=\operatorname{limind}_{\alpha \downarrow 0} \mathcal{O}\left(D_{\alpha}\right)
$$

$\left(\varphi_{j}\right)$ est totale dans $\mathcal{O}(D)$, d'après le théorème 2.1. Puisque $\mathcal{O}(D)$ est partout dense dans $\mathcal{O}\left(D_{\alpha}\right),\left(\varphi_{j}\right)$ est aussi totale dans $\mathcal{O}\left(D_{\alpha}\right)$. Il en résulte que pour tout $f \in \mathcal{O}\left(D_{\alpha}\right)$, sa restriction à $K$ est un élément de l'espace hilbertien $H_{0}$. 
Soit $f \in \mathcal{O}\left(D_{\alpha}\right)$. Comme élément de $H_{0}, f$ est la somme de la série $\sum_{0}^{\infty} \varphi_{j}^{\prime}(f) \varphi_{j}$, où

$$
\varphi_{j}^{\prime}(f)=\int_{K} f \varphi_{j} d \mu_{0}
$$

Soit $\beta<\alpha, \varepsilon>0$. On va montrer que

$$
\left|\varphi_{j}^{\prime}(f)\right| \leq C(\beta, \varepsilon)\|f\|_{\beta} \gamma_{j}^{-\beta+\varepsilon}, \quad \forall j .
$$

En effet, il est facile de voir que $\left\|\varphi_{j}^{\prime}\right\|_{1}^{*}=\gamma_{j}^{-1},\left\|\varphi_{j}^{\prime}\right\|_{0}^{*}=1$, ce qui donne, avec (3.3),

$$
\left\|\varphi_{j}^{\prime}\right\|_{\beta}^{*} \leq C(\beta, \varepsilon) \gamma_{j}^{-\beta+\varepsilon}, \quad \forall j .
$$

d'autre part, $\mathcal{O}(D)$ est partout dense dans $\mathcal{O}\left(D_{\alpha}\right)$ et $B_{\beta} \Subset D_{\alpha}$; on a donc

$$
\left\|\varphi_{j}^{\prime}\right\|_{\beta}^{*}=\sup \left\{\left|\varphi_{j}^{\prime}(g)\right|: g \in \mathcal{O}\left(D_{\alpha}\right),\|g\|_{\beta} \leq 1\right\}
$$

d'où (4.1).

(2.17) et (4.1) assurent la convergence normale de la série $\sum_{j=0}^{\infty} \varphi_{j}^{\prime}(f) \varphi_{j}$ sur tout compact de $D_{\alpha}$. D'après la proposition 1.2, la somme de cette série est égale à $f \operatorname{sur} D_{\alpha}$.

2) Supposons $\sum c_{j} \varphi_{j}$ convergente dans $\mathcal{O}\left(D_{\alpha}\right)$, soit $f$ sa somme. Alors $c_{j}=$ $\varphi_{j}^{\prime}(f)$ et (ii) est une conséquence immédiate de (4.1).

3) C'est une conséquence facile de (2.17) et de (4.1) appliquée à $f=\varphi_{j}$, avec $\varphi_{j}^{\prime}\left(\varphi_{j}\right)=1$.

4) Soit

$$
u=\operatorname{Reg} \sup \left(\limsup _{j \rightarrow \infty} \frac{\log \left|\varphi_{j}\right|}{\log \gamma_{j}}\right) .
$$

En raison de (2.13) il suffit de prouver que l'inégalité $u(z)>\omega(z, K, D)$ est impossible, $\forall z \in D \backslash \widehat{K}_{D}$. Supposons-la vérifié en un point $a \in D \backslash \widehat{K}_{D}$ et soit $\alpha:=\omega(a, K, D)$. A l'aide du lemme de Hartogs on voit facilement qu'il existe une boule ouverte $B \subset D$ de centre $a, C>0$ et $\beta<\alpha$ tels que

$$
\left|\varphi_{j}\right|_{B} \leq C \gamma_{j}^{\beta}, \quad \forall j .
$$

Soit $f=\sum \varphi_{j}^{\prime}(f) \varphi_{j}$ une fonction holomorphe admettant $D_{\alpha}$ comme domaine d'holomorphie. Les inégalités (4.1), (4.2) et (2.17) montrent que la série $\sum \varphi_{j}^{\prime}(f) \varphi_{j}$ converge dans $\mathcal{O}\left(D_{\alpha} \cup B\right), f$ est donc analytiquement prolongeable à $D_{\alpha} \cup B$ : c'est impossible.

R e marque. L'existence de bases communes est démontrée par Zakharyuta ([Za,1]) dans le cas particulier où $D$ est "très fortement pseudoconvexe" par une méthode différente de celle présentée ici (voir [Ze,1]). L'assertion 4 du théorème est due à Nguyen et Siciak dans un cas particulier (voir [N-S]). 


\section{Application 1 - Généralisation du théorème de Whittaker}

THÉORÈme 4.2. Soit $(K, D)$ un condensateur régulier dans $\mathbb{C}^{n}$. Si $\left(f_{i}\right)$ est une base commune des espaces $\mathcal{O}(D)$ et $\mathcal{O}\left(\widehat{K}_{D}\right)$, alors elle est une base commune des espaces $\mathcal{O}\left(D_{\alpha}\right), 0<\alpha<1$.

Dém onstration. La première preuve de cet énoncé figure dans [Za1]. Nous en donnons brièvement ici une nouvelle due à Lassere $[\mathrm{L}]$. C'est une conséquence facile de l'assertion suivante : Soit $\mathcal{C}$ une partie équicontinue de $\mathcal{L}(\mathcal{O}(K), \mathcal{O}(K))$. Si sa restriction à $\mathcal{O}(D)$ est une partie équicontinue de $\mathcal{L}(\mathcal{O}(D), \mathcal{O}(D))$, alors sa restriction à $\mathcal{O}\left(D_{\alpha}\right)$ est une partie équicontinue de $\left.\mathcal{L}\left(\mathcal{O}\left(D_{\alpha}\right), \mathcal{O}\left(D_{\alpha}\right)\right), \forall \alpha \in\right] 0,1[$.

Cette assertion se démontre élémentairement à l'aide de la base $\left(\varphi_{j}\right)$.

\section{Application 2 - Propriété produit de la fonction extrémale}

ThÉORÈme 4.3. Pour $j=1, \ldots, p$ soit $D_{j}$ un ouvert pseudoconvexe de $\mathbb{C}^{n j}$ et $E_{j}$ un sous-ensemble $\mathcal{K}$-analytique de $D_{j}$. Alors pour tout $z=\left(z_{1}, \ldots, z_{p}\right) \in$ $D_{1} \times \ldots \times D_{p}$ on $a$

$$
\omega\left(z, E_{1} \times \ldots \times E_{p}, D_{1} \times \ldots \times D_{p}\right)=\max _{j} \omega\left(z_{j}, E_{j}, D_{j}\right) .
$$

Démonstration. C'est une conséquence du Théorème 4.1.4, pour les détails voir $[\mathrm{N}-\mathrm{S}]$.

5. Isomorphisme d'espaces de fonctions holomorphes. Rappelons quelques résultats de la théorie des échelles hilbertiennes de Mityagin dans le contexte où nous allons les appliquer.

Soit $\left(a_{j}\right)$ une suite croissante de réels $>0$ telle que $\lim _{j \rightarrow \infty} a_{j}=\infty$; on note

$$
l^{2}\left(\exp \left(\lambda a_{j}\right)\right):=\left\{\xi=\left(\xi_{j}\right): \sum_{j=1}^{\infty}\left|\xi_{j}\right|^{2} \exp \left(2 \lambda a_{j}\right)<\infty\right\} .
$$

C'est un espace de Hilbert avec la norme $\|\xi\|_{\lambda}^{2}:=\sum_{j=1}^{\infty}\left|\xi_{j}\right|^{2} \exp \left(2 \lambda a_{j}\right)$.

Pour tout $\alpha \in \mathbb{R}$, on définit deux espaces :

$$
\begin{array}{ll}
L_{\alpha}^{2}\left(a_{j}\right)=\bigcap_{\lambda<\alpha} l^{2}\left(\exp \left(\lambda a_{j}\right)\right), & -\infty<\alpha<\infty, \\
\bar{L}_{\alpha}^{2}\left(a_{j}\right)=\bigcup_{\lambda>\alpha} l^{2}\left(\exp \left(\lambda a_{j}\right)\right), & -\infty<\alpha<\infty .
\end{array}
$$

Le premier espace est une limite projective, c'est un espace de Fréchet; le deuxième est une limite inductive.

Les cas qui nous intéressent vérifient la propriété suivante :

$$
\sum_{j=1}^{\infty} e^{-t a_{j}}<\infty \quad \forall t>0
$$

C'est la condition de nucléarité des espaces (5.1) et (5.2). 
Le théorème d'isomorphisme de Mityagin s'exprime dans ce cas particulier de la façon suivante $([\mathrm{Mi}])$ :

Soient $\left(a_{j}\right)$ et $\left(b_{j}\right)$ deux suites croissantes de réels $>0$ vérifiant (5.3). Alors les conditions suivantes sont équivalentes :

$$
\begin{gathered}
L_{\alpha}^{2}\left(a_{j}\right) \simeq L_{\alpha}^{2}\left(b_{j}\right) . \\
0<\liminf _{j \rightarrow \infty} \frac{a_{j}}{b_{j}} \leq \limsup _{j \rightarrow \infty} \frac{a_{j}}{b_{j}}<\infty . \\
\bar{L}_{\alpha}^{2}\left(a_{j}\right) \simeq \bar{L}_{\alpha}^{2}\left(b_{j}\right) .
\end{gathered}
$$

Soit $\Delta^{n}$ (resp. $\bar{\Delta}^{n}$ ) le polydisque unité ouvert (resp. fermé). Soit $v: \mathbb{N}^{*} \rightarrow \mathbb{N}^{n}$ une bijection telle que $|v(j)| \leq|v(j+1)|, \forall j \in \mathbb{N}^{n}$. Posons $e_{j}(z)=z^{v(j)}$ et $v_{j}=|v(j)|$ pour $j \in \mathbb{N}^{*}$. Il est facile de voir que $v_{j} \sim j^{1 / n}$ lorsque $j \rightarrow \infty$. Il résulte alors des inégalités de Cauchy que l'application linéaire

$$
\mathcal{O}\left(e^{\alpha} \Delta^{n}\right) \rightarrow L_{\alpha}^{2}\left(j^{1 / n}\right), \quad f=\sum_{j=1}^{\infty} \xi_{j} e_{j} \mapsto \xi=\left(\xi_{j}\right),
$$

est un isomorphisme pour tout $\alpha \in \mathbb{R}$ qui induit également un isomorphisme de $\mathcal{O}\left(e^{\alpha} \bar{\Delta}^{n}\right)$ sur $\bar{L}_{\alpha}^{2}\left(j^{1 / n}\right)$.

Les résultats du $\S 4$ peuvent également s'interpréter en terme d'isomorphisme. En effet, les estimations (4.9) et (4.10) impliquent que l'application

$$
\mathcal{O}\left(D_{\alpha}\right) \rightarrow L_{\alpha}^{2}\left(\log \gamma_{j}\right), \quad f=\sum_{j=1}^{\infty} \xi_{j} \varphi_{j} \mapsto \xi=\left(\xi_{j}\right),
$$

est un isomorphisme pour tout $\alpha \in] 0,1]$ qui induit un isomorphisme de $\mathcal{O}\left(K_{\alpha}\right)$ sur $\bar{L}_{\alpha}^{2}\left(\log \gamma_{j}\right)$ où $\gamma_{j}=\gamma_{j}(K, D)$. Nous allons prouver le résultat suivant :

ThÉORÈme 5.1. Soit $(K, D)$ un condensateur P-régulier. Alors on a les propriétés suivantes:

$$
\begin{aligned}
0<\liminf _{j \rightarrow \infty} \frac{\log \gamma_{j}}{j^{1 / n}} & \leq \limsup _{j \rightarrow \infty} \frac{\log \gamma_{j}}{j^{1 / n}}<\infty, \\
\mathcal{O}(D) & \simeq \mathcal{O}\left(\Delta^{n}\right) \\
\mathcal{O}(K) & \simeq \mathcal{O}\left(\bar{\Delta}^{n}\right) .
\end{aligned}
$$

Démonstration. La relation (1) est équivalente à (2) grâce aux isomorphismes (5.5), (5.6) via le théorème d'isomorphisme de Mityagin.

Pour démontrer (2), on peut supposer $D$ convexe (voir [Mi-He]). Soit $E$ un polydisque fermé tel que $E \subset D$. Alors $(E, D)$ est un condensateur régulier tel que $\widehat{E}_{D}=E$. Donc d'après $(5.6), \mathcal{O}(E) \simeq \bar{L}_{0}^{2}\left(\log \gamma_{j}^{\prime}\right)$ où $\gamma_{j}^{\prime}=\gamma_{j}(E, D)$. D'après (5.4) et (5.5), on a

$$
0<\liminf _{j \rightarrow \infty} \frac{\log \gamma_{j}^{\prime}}{j^{1 / n}} \leq \limsup _{j \rightarrow \infty} \frac{\log \gamma_{j}^{\prime}}{j^{1 / n}}<\infty .
$$


Puisque $\mathcal{O}(D) \simeq L_{1}^{2}\left(\log \gamma_{j}^{\prime}\right)$, il résulte du théorème d'isomorphisme de Mityagin que $\mathcal{O}(D) \simeq \mathcal{O}\left(\Delta^{n}\right)$. D'où l'isomorphisme (2). Les estimations (1) en résultent grâce au théorème de Mityagin ([Mi]).

L'isomorphisme (3) en résulte par le même théorème puisque $\mathcal{O}(K) \simeq$ $\bar{L}_{0}^{2}\left(\log \gamma_{j}\right)$.

Remarque. L'isomorphisme (2) du théorème 5.1 est dû à Zakharyuta $([\mathrm{Za}, 1])$ dans le cas particulier où $D$ est "très fortement pseudoconvexe". Le cas général est dû à Aytuna ([A]) et a également été annoncé par Zakharyuta $([\mathrm{Za}, 3])$. L'isomorphisme (3) du théorème 5.1 est dû à Zakharyuta ([Za,1], [Za,3]). La démonstration présentée ici semble plus élémentaire.

6. S.D.O.B. et fonctions séparément analytiques. Pour $j=1, \ldots, p$, soient $D_{j}$ un ouvert borné de $\mathbb{C}^{n_{j}}$ et $E_{j}$ un sous-ensemble non $\mathbb{C}_{j}^{n}$-pluripolaire de $D_{j}$. On pose

$$
\begin{aligned}
X:= & \left(D_{1} \times E_{2} \times \ldots \times E_{p}\right) \cup\left(E_{1} \times D_{2} \times E_{3} \times \ldots \times E_{p}\right) \\
& \cup\left(E_{1} \times \ldots \times E_{p-1} \times D_{p}\right), \\
\widehat{X}:= & \left\{\left(z_{1}, \ldots, z_{p}\right) \in D_{1} \times \ldots \times D_{p}: \sum_{j=1}^{p} \omega\left(z_{j}, E_{j}, D_{j}\right)<1\right\} .
\end{aligned}
$$

Une fonction $f\left(z_{1}, \ldots, z_{p}\right)$ définie sur $X$ est dite séparément analytique sur $X$ lorsque pour tout $k=1, \ldots, p$ et pour $z \in E_{1} \times \ldots \times \check{E}_{k} \times \ldots \times E_{p}\left(\check{E}_{k}\right.$ signifie que $E_{k}$ n'y figure pas), la fonction $\xi \rightarrow f\left(z_{1}, \ldots, z_{k-1}, \xi, z_{k+1}, \ldots, z_{p}\right)$ est analytique sur $D_{k}$.

THÉORÈME 6.1. On suppose que les $E_{j}$ sont $\mathcal{K}$-analytiques sauf au plus un, et que les ouverts bornés $D_{j}$ correspondants sont pseudoconvexes. Alors pour toute fonction $f$ séparément analytique sur $X$, il existe une et une seule fonction $\widehat{f}$ analytique sur $\widehat{X}$ telle que $f=\widehat{f}$ sur $X \cap \widehat{X}$.

La démonstration est divisée en plusieurs étapes :

1) Existence de $\widehat{f}$, cas $p=2$. Ce cas $p=2$ a été traité dans [N-Z,2]. On résume ici la preuve. On peut supposer $D:=D_{1}$ pseudoconvexe et $E:=E_{1} \mathcal{K}$-analytique. On pose $G:=D_{2}, F:=E_{2}$.

On se ramène au cas où $E$ est compact et $f$ continue et bornée sur $E \times G$. Sous ces conditions, on choisit une suite croissante $\left(D_{s}\right)$ d'ouverts hyperconvexes telle que $E \subset D_{s} \Subset D$ et $\bigcup D_{s}=D$. Pour $s$ fixé, soit $\left(\varphi_{j}\right)$ le S.D.O.B. associé au condensateur $\widetilde{E}:=E \cap \widetilde{D}_{s}$.

Pour $w \in F, f(\cdot, w)$ est holomorphe sur $D$, donc c'est un élément de $\mathcal{O}^{2}\left(\widetilde{D}_{s}, d \mu_{1}\right)$ et peut s'écrire $f(\cdot, w)=\sum_{j=0}^{\infty} C_{j}(w) \varphi_{j}$, avec

$$
C_{j}(w)=\int_{\tilde{E}} f(z, w) \bar{\varphi}_{j}(z) d \mu_{0}(z), \quad \mu_{0}=\left(d d^{c} \omega\left(\cdot, \widetilde{E}, \widetilde{D}_{s}\right)\right)^{n_{1}} .
$$


L'intégrale définit une fonction analytique sur $G$ que l'on note encore par $C_{j}$. Par des majorations adéquates utilisant (2.12) et (2.13), on montre que la série $\sum_{j=0}^{\infty} C_{j}(w) \varphi_{j}(z)$ converge uniformément sur tout compact de l'ouvert

$$
\begin{aligned}
X_{s} & :=\left\{(z, w) \in \widetilde{D}_{s} \times G: \omega\left(z, \widetilde{E}, \widetilde{D}_{s}\right)+\omega(w, F, G)<1\right\} \\
& =\left\{(z, w) \in D_{s} \times G: \omega\left(z, E, D_{s}\right)+\omega(w, F, G)<1\right\} .
\end{aligned}
$$

Soit $F_{s}(z, w)$ la somme de cette série. Elle est évidemment égale à $f(z, w)$ sur $X_{s} \cap\left(\widetilde{D}_{s} \times F\right)=X_{s} \cap\left(D_{s} \times F\right)$, on vérifie qu'elle l'est encore sur $X_{s} \cap\left(\widetilde{E}_{s} \times G\right)=$ $X_{s} \cap(E \times G)$. On vérifie ensuite que $F_{s}=F_{s+1}$ sur $X_{s}$. Ces vérifications utilisent (1.4bis). On obtient $\widehat{f}$ en "recollant les morceaux" $\left(X_{s}, F_{s}\right)$ et en remarquant que $\bigcup X_{s}=\widehat{X}$.

2) Existence de $\widehat{f}$, cas $p>2$. On a besoin de quelques nouvelles notations. Pour $\alpha=\left(\alpha_{1}, \ldots, \alpha_{p}\right) \in\left(\mathbb{R}_{*}^{+}\right)^{p}$, on pose

$$
\begin{aligned}
D_{j}^{\alpha_{j}} & :=\left\{\xi \in D_{j}: \omega\left(\xi, E_{j}, D_{j}\right)<\alpha_{j}\right\}, & D^{\alpha}: & =D_{1}^{\alpha_{1}} \times \ldots \times D_{p}^{\alpha_{p}}, \\
E_{j}^{\alpha_{j}} & :=E_{j} \cap D_{j}^{\alpha_{j}}, & E^{\alpha} & =E_{1}^{\alpha_{1}} \times \ldots \times E_{p}^{\alpha_{p}} .
\end{aligned}
$$

On peut supposer $E_{1}, \ldots, E_{p-1} \mathcal{K}$-analytiques et $D_{1}, \ldots, D_{p-1}$ pseudoconvexes. On raisonne par récurrence en supposant que l'énoncé est vrai pout $p-1$.

(i) Soit $T_{p}$ le simplexe $\left\{\left(x_{1}, \ldots, x_{p}\right) \in\left(\mathbb{R}_{*}^{+}\right)^{p}: \sum_{j=1}^{p} x_{j} \leq 1\right\}$. On va montrer que pour tout $\alpha \in T_{p}$ il existe une fonction $f_{\alpha}$ analytique sur $D^{\alpha}$ telle que $f_{\alpha}=f \operatorname{sur} D^{\alpha} \cap X$. On pose $\theta_{j}=\alpha_{j}\left(1-\alpha_{p}\right)^{-1}$ pour $j=1, \ldots, p-1, D^{\theta}=$ $D_{1}^{\theta_{1}} \times \ldots \times D_{p-1}^{\theta_{p-1}}, E^{\theta}=E_{1}^{\theta_{1}} \times \ldots \times E_{p-1}^{\theta_{p-1}}$. Puisque $f$ est séparément analytique sur $X, f(z, \cdot)$ est analytique sur $D_{p}$ pour $z \in E^{\theta}$, pour $w \in F, f(\cdot, w)$ est séparément analytique sur

$$
X_{*}:=\left(D_{1} \times E_{2} \times \ldots \times E_{p-1}\right) \cup \ldots \cup\left(E_{1} \times \ldots \times E_{p-2} \times D_{p-1}\right) .
$$

Donc, par hypothèse de récurrence, il existe une fonction $\widehat{f}_{w}$ analytique sur $\widehat{X}_{*}$ telle que $\widehat{f}_{w}=f(\cdot, w)$ sur $\widehat{X}_{*} \cap X_{*}$. On remarque que $D^{\theta} \subset \widehat{X}_{*}$, car $\sum_{j=1}^{p-1} \theta_{j} \leq 1$. On considère sur

$$
Y:=\left(D^{\theta} \times E_{p}\right) \cup\left(E^{\theta} \times D_{p}\right)
$$

la fonction $\tilde{f}$,

$$
\widetilde{f}(z, w)= \begin{cases}f(z, w) & \text { si }(z, w) \in E^{\theta} \times D_{p}, \\ \widehat{f}_{w}(z) & \text { si }(z, w) \in D^{\theta} \times E_{p} .\end{cases}
$$

$\tilde{f}$ est séparément analytique sur $Y$ et égale à $f$ sur $Y \cap X$, donc d'après le cas $p=2$ il existe une fonction $g$ analytique sur

$$
\widehat{Y}=\left\{(z, w) \in D^{\theta} \times D_{p}: \omega\left(z, E^{\theta}, D^{\theta}\right)+\omega\left(w, E_{p}, D_{0}\right)<1\right\}
$$

telle que $g=\tilde{f}$ sur $\widehat{Y} \cap X$. 
La propriété produit de la fonction extrémale (4.3) et (1.4) donnent, pour $z=\left(z_{1}, \ldots, z_{p-1}\right) \in D^{\theta}$,

$$
\omega\left(z, E^{\theta}, D^{\theta}\right)=\max _{1 \leq j \leq p-1} \frac{1-\alpha_{p}}{\alpha_{j}} \omega\left(z_{j}, E_{j}, D_{j}\right) .
$$

Pour $(z, W) \in D^{\alpha} \subset D^{\theta} \times D_{p}^{\alpha_{p}}$ on a

$$
\omega\left(z, E^{\theta}, D^{\theta}\right)+\omega\left(w, E_{p}, D_{0}\right)<1-\alpha_{p}+\alpha_{p}=1,
$$

donc $D^{\alpha} \subset \widehat{Y}$. On remarque que $f=g$ sur $D^{\alpha} \cap X$; en effet, $\widetilde{f}=f=g$ sur $\widehat{Y} \cap Y \cap X$ qui contient $D^{\alpha} \cap X$ car $D^{\alpha} \subset \widehat{T}$ et $D^{\alpha} \cap X \subset Y \cap X$. On obtent donc le résultat annoncé en posant $f_{\alpha}=g$.

(ii) Terminons maintenant la preuve de l'existence de $\widehat{f}$. Soient $\alpha, \beta \in T_{p}$, soient $f_{\alpha}$ et $f_{\beta}$ comme dans (i). Alors $f_{\alpha}=f_{\beta}$ sur $D^{\alpha} \cap D^{\beta} \cap X$ qui contient $E^{\gamma}$, avec $\gamma=\left(\min \left(\alpha_{1}, \beta_{1}\right), \ldots, \min \left(\alpha_{p}, \beta_{p}\right)\right)$. Or d'après (1.4bis), $E^{\gamma}$ est "localement" non pluripolaire dans $D^{\gamma}=D^{\alpha} \cap D^{\omega}$, donc $f_{\alpha}=f_{\beta}$ sur $D^{\alpha} \cap D^{\beta}$. On peut donc recoller les morceaux $\left(D^{\alpha}, f_{\alpha}\right)$ pour tous les $\alpha \in T_{p}$ : on obtient une fonction $\widehat{f}$ analytique sur

$$
\widehat{X}=\bigcup_{\alpha \in T_{p}} D^{\alpha}
$$

et égale à $f$ sur $\widehat{Y} \cap X$.

3) Unicité. Si $g$ est analytique sur $\widehat{X}$ et égale à $f$ sur $\widehat{X} \cap X$, alors pour tout $\alpha \in T_{p}, \widehat{f}$ et $g$ sont analytiques sur $D^{\alpha} \subset \widehat{X}$ et égales sur $E^{\alpha}$ qui est "localement" non pluripolaire dans $D^{\alpha}$, donc identiques sur $D^{\alpha}$. Par conséquent $\widehat{f}=g$ sur $\widehat{X}$, réunion des $D^{\alpha}$.

R e marques. 1) Le théorème 5.1 est une extension du théorème bien connu de J. Siciak $([\mathrm{Si}, 1])$.

2) Dans le cas $p=2$, l'hypothèse que $D=D_{1}$ et $G=D_{2}$ sont bornés, oubliée dans [N-Z,2], est faite pour garantir les propriétés de convergence de la fonction extrémale. Plus précisément, si $D$ est un ouvert borné de $\mathbb{C}^{n}$, alors :

- pour toute suite croissante $\left(E_{s}\right)$ de sous-ensembles de $\mathbb{C}^{n}$,

$$
\omega\left(z, E_{s}, D\right) \searrow \omega\left(z, \bigcup E_{s}, D\right), \quad \forall z \in D,
$$

- pour toute suite croissante $\left(D_{s}\right)$ d'ouverts de $\mathbb{C}^{n}$ telle que $\bigcup D_{s}=D$ et tout $E \subset \mathbb{C}^{n}$,

$$
\omega\left(z, E_{s}, D\right) \searrow \omega(z, E, D), \quad \forall z \in D .
$$

Lorsque $D$ et $G$ ne sont pas bornés ( $D$ ouvert pseudoconvexe, $G$ ouvert), la démonstration de $[\mathrm{N}-\mathrm{Z}, 2]$ est valable sous les hypothèses suivantes : 
- Il existe une suite croissante d'ouverts hyperconvexes $\left(D_{t}\right)$ telle que $D_{t} \Subset D$, $\bigcup D_{t}=D$ et que

$$
\begin{aligned}
& \lim _{t \rightarrow \infty} \omega\left(z, E \cap D_{s}, D_{t}\right)=\omega\left(z, E \cap D_{s}, D\right), \quad \forall z \in D, \forall s, \\
& \lim _{s \rightarrow \infty} \omega\left(z, E \cap D_{s}, D\right)=\omega(z, E, D), \quad \forall z \in D .
\end{aligned}
$$

- Il existe une suite croissante d'ouverts $\left(G_{t}\right)$ ayant les mêmes propriétés par rapport au couple $(F, G)$.

Ces hypothèses sont satisfaites lorsque $E$ et $F$ sont des compacts $P$-réguliers dans $D$ et $G$ respectivement, avec $D, G$ pseudoconvexes : on retrouve le résultat de Zakharyuta [Za,2].

Elles sont également satisfaites lorsque $D$ et $G$ sont hyperconvexes ([K]).

3) Dans son article de synthèse sur les fonctions plurisousharmoniques [Sa], A. Sadullaev a donné brièvement une autre démonstration du théorème 5.1 avec $p=2, D_{i}$ pseudoconvexe et $E_{i}$ borélien $(i=1,2)$.

Note. V. P. Zakharyuta nous a aimablement communiqué son texte polycopié "Espaces de fonctions analytiques" (95 pages en Russe). On y trouve des énoncés semblables à certains résultats de notre texte avec des techniques de démonstrations différentes.

\section{Bibliographie}

[A] A. Aytuna, On Stein manifolds $M$ for which $\mathcal{O}(M)$ is isomorphic to $\mathcal{O}\left(\Delta^{n}\right)$ as Fréchet spaces, Manuscripta Math. 62 (1988), 297-315.

[B-T] E. Bedford and B. A. Taylor, A new capacity for plurisubharmonic functions, Acta Math. 149 (1982), 1-41.

[Be] S. Bergman, The Kernel Function and Conformal Mapping, Math. Surveys 5, Amer. Math. Soc., 1950.

[H-M] G. Henkin and B. Mityagin, Linear problems of complex analysis, Russian Math. Surveys 26 (1972), 99-164

Ho] L. Hörmander, An Introduction to Complex Analysis in Several Variables, NorthHolland, 1973.

[K] M. Klimek, Pluripotential Theory, Oxford University Press, 1991.

[L] P. Lassere, Interpolation d'opérateurs entre espaces de fonctions holomorphes, Ann. Polon. Math. 56 (1991), 97-102.

[M] B. Mityagin, Approximate dimensions and bases in nuclear spaces, Russian Math Surveys 16 (1961), 59-127.

[N-S] T. V. Nguyen et J. Siciak, Fonctions plurisousharmoniques extrémales et systèmes doublement orthogonaux de fonctions holomorphes, Bull. Sci. Math. (2) 115 (1991) 235-244

[N-Z,1] T. V. Nguyen et A. Zeriahi, Familles de polynômes presque partout bornées, Bull. Sci. Math. (2) 107 (1983), 81-91.

[N-Z,2] -, 一, Une extension du théorème de Hartogs sur les fonctions séparément analytiques, dans: Analyse complexe multivariable, Récents Développements, A. Meril (éd.), Editel, Rende, 1991, 183-194. 
[R-N] F. Riesz et B. Sz. Nagy, Leçons d'Analyse Fonctionnelle, Académie des Sciences de Hongrie, 1952

[Sa] A. Sadullaev, Fonctions plurisousharmoniques, dans : Progrès en Mathématiques, Itogi Nauki 8, 274 (1985), 65-113 (en russe).

[Si,1] J. Siciak, Separately analytic functions and envelopes of holomorphy of some lower dimensional subsets of $\mathbb{C}^{n}$, Ann. Polon. Math. 22 (1969), 145-171.

$[\mathrm{Si}, 2] \quad-$ Extremal plurisubharmonic functions in $\mathbb{C}^{n}$, ibid. 39 (1981), 175-211.

[V] D. Vogt, Eine Charakterisierung der Potenzreihenräume von endlichem Typ und ihre Folgerungen, Manuscripta Math. 37 (1982), 269-301.

[Za,1] V. P. Zakharyuta, Fonctions plurisousharmoniques, échelles hilbertiennes et isomorphismes d'espaces de fonctions analytiques de plusieurs variables, I et II, Teor. Funktsiı Funktsional. Anal. i Prilozhen. 19 (1974), 133-157, et 21 (1974), 65-83 (en russe).

[Za,2] -, Separately analytic functions, generalizations of Hartog's theorem and envelopes of holomorphy, Math. USSR-Sb. 30 (1976), 51-67.

[Za,3] -, Isomorphisms of spaces of analytic functions, Soviet Math. Dokl. 22 (1980), 631-634.

[Ze,1] A. Zeriahi, Bases de Schauder et isomorphismes d'espaces de fonctions holomorphes, C. R. Acad. Sci. Paris 310 (1990), 691-694.

$[\mathrm{Ze}, 2] \quad$ - Fonction de Green pluricomplexe à pôle à l'infini sur un espace de Stein parabolique et applications, Math. Scand. 69 (1991), 89-126. 